\title{
Giant Spin Relaxation of an Ultracold Cesium Gas
}

\author{
J. Söding, D. Guéry-Odelin, P. Desbiolles, G. Ferrari,* and J. Dalibard \\ Laboratoire Kastler Brossel, ${ }^{\dagger}$ Ecole Normale Supérieure, 24 rue Lhomond, F-75231 Paris CEDEX 05, France.
} (Received 6 November 1997)

\begin{abstract}
We have measured the rate of inelastic collisions in a cloud of doubly polarized ground-state cesium atoms $\left(F=m_{F}=4\right)$ confined in a magnetic trap for temperatures $T$ between 8 and $70 \mu \mathrm{K}$. We find a two-body rate coefficient varying as $T^{-0.63}$. At $8 \mu \mathrm{K}$ it reaches $4 \times 10^{-12} \mathrm{~cm}^{3} \mathrm{~s}^{-1}$ which is 3 orders of magnitude larger than predicted, ruling out a Bose-Einstein condensation of $\mathrm{Cs}$ in this internal state. [S0031-9007(98)05435-0]
\end{abstract}

PACS numbers: 34.50.Pi, 03.75.Fi, 05.30.Jp, 32.80.Pj

The recent success of Bose-Einstein condensation experiments with atomic vapors of rubidium [1], lithium [2], and sodium [3] has attracted a lot of attention. The atomic sample is spin polarized and confined in a magnetic trap. The condensation is reached using the elastic collisions between the trapped atoms to evaporatively cool the gas to a temperature below $1 \mu \mathrm{K}$. Although these gaseous assemblies are not truly stable at such a low temperature, the time involved with the relaxation processes, such as two-body spin dipolar relaxation or three-body recombination, is quite long. For typical atomic densities of $10^{13}-10^{14} \mathrm{~cm}^{-3}$, the lifetime for the condensate is several seconds, leaving enough time for a study of the condensate properties.

We show in this Letter that this metastability is not "universal": For a gas of doubly polarized cesium atoms, inelastic collisions limit the atomic density to a value much below the condensation threshold. The case of cesium is of particular interest because the hyperfine splitting $\Delta E \simeq h \times 9.2 \mathrm{GHz}$ of its ground state into two sublevels with angular momenta $F=3$ and $F=4$ is the basis of primary time and frequency standards.

A cesium atomic gas in its doubly polarized (electron + nucleus) ground state $\left(F=m_{F}=4\right)$, trapped in a magnetic field minimum, was initially considered to be a very good candidate for Bose-Einstein condensation (BEC) [4]. In zero magnetic field and for densities below $10^{13} \mathrm{~cm}^{-3}$, the dominant loss process is predicted to be a spin dipole relaxation, occurring through the magnetic dipole-dipole interaction. After a collision of two polarized cesium atoms, one or both atoms may emerge in the level $F=3$. The internal energy ( $\Delta E$ or $2 \Delta E$ ) is converted into kinetic energy shared between the two atoms. As $\Delta E=$ $k_{B} \times 0.44 \mathrm{~K}$ is much larger than the typical trap depth $\left(\sim k_{B} \times 1 \mathrm{mK}\right)$, the two atoms escape from the trap after the inelastic collision. The rate coefficient for this process was estimated to be in the range of $10^{-15} \mathrm{~cm}^{3} \mathrm{~s}^{-1}$ [4], which should not be a limitation for the achievement of BEC with densities similar to other alkali experiments.

We report here measurements performed on polarized Cs atoms trapped in a static magnetic trap [5]. The cloud is prepared with adjustable temperature using evaporative cooling. We monitor the decay of atoms out of the trap as a function of time. At $8 \mu \mathrm{K}$ we find a two-body loss rate of $4 \times 10^{-12} \mathrm{~cm}^{3} \mathrm{~s}^{-1}$. This spectacular deviation from the theoretical expectation by 3 orders of magnitude may be a consequence of the zero-energy resonance for elastic collisions that has recently been observed in cesium [6].

The experiment is performed in a double cell system which has been described in detail elsewhere [7]. The experimental sequence is the following: During the first $5 \mathrm{~s}$, we collect $\sim 2 \times 10^{8}$ atoms in a magneto-optical trap (MOT) in the upper cell from the residual ${ }^{133} \mathrm{Cs}$ vapor (pressure $\sim 10^{-8}$ mbar). The trap light and the magnetic field are then switched off, and the atoms fall into the lower cell located $70 \mathrm{~cm}$ below (pressure $<10^{-10}$ mbar). They are recaptured in a second MOT, cooled by a $20 \mathrm{~ms}$ molasses phase, and transferred into the magnetic trap.

This magnetic trap is of the Ioffe-Pritchard type, i.e., it is purely static and it consists of a nonzero local minimum of the modulus of the magnetic field. The field is generated by three identical circular coils whose axes point towards $+x,-x$, and $+y$, respectively ( $z$ denotes the vertical axis), and whose centers are at equal distance $l=40 \mathrm{~mm}$ from the center $O$ of the trap (Fig. 1). Each coil has 80 turns with an average diameter of $34 \mathrm{~mm}$. The quadrupole MOT field is generated with the $+x-x$ pair and leads to trapping around $O$. At the end of the molasses phase, the atoms are optically pumped into the state $F=m_{F}=4$ (quantization axis along $y$ ) by a $100 \mu$ s laser pulse.
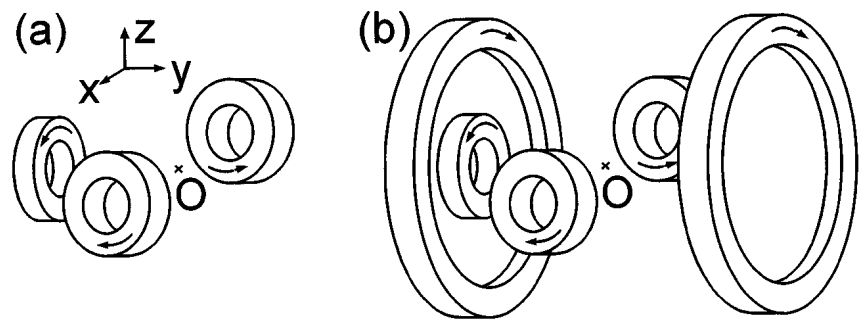

FIG. 1. (a) Three-coil configuration for the magnetic trap (analogous to a "baseball trap"). The two opposite coils are also used for the MOT. (b) Two extra Helmholtz coils serve for the compression of the atomic cloud in the $x z$ plane. 
With the same current running through the three coils, the resulting field has a local minimum in $O$ which ensures that the atomic cloud from the MOT is centered in the magnetic trap. The leading terms in the magnetic field variation around $O$ are $\left(b^{\prime} x, B_{0}+b^{\prime \prime} y^{2} / 2,-b^{\prime} z\right)$. For a 51-A current we get $B_{0}=11 \mathrm{mT}, b^{\prime}=1.1 \mathrm{~T} / \mathrm{m}$, and $b^{\prime \prime}=$ $60 \mathrm{~T} / \mathrm{m}^{2}$. Near its center the trap is nearly harmonic and has oscillation frequencies $\omega_{x}=\omega_{z}=\left(\mu b^{12} / m B_{0}\right)^{1 / 2}=$ $2 \pi \times 11 \mathrm{~Hz}$ and $\omega_{y}=\left(\mu b^{\prime \prime} / m\right)^{1 / 2}=2 \pi \times 8 \mathrm{~Hz}$, where $\mu$ is the Bohr magneton and $m$ the atomic mass. The ratio between velocity and position widths in the MOT, $\Delta v / \Delta x \approx 2 \pi \times 10 \mathrm{~Hz}$, is close to $\omega_{x, z}$ and $\omega_{y}$, which ensures a minimum loss in phase space density during the transfer to the magnetic trap.

Once magnetically trapped, the atomic cloud is further compressed by reducing $B_{0}$ to $0.15 \mathrm{mT}$ using a pair of Helmholtz coils aligned with the $y$ axis (Fig. 1). The transverse $(x-z)$ oscillation frequencies increase to $90 \mathrm{~Hz}$ while the longitudinal $(y)$ frequency remains unchanged. At this stage the cigar-shaped atomic cloud contains $N \approx 5 \times 10^{7}$ atoms at $100 \mu \mathrm{K}$ and has a peak density $6 \times 10^{10} \mathrm{~cm}^{-3}$.

We then perform a forced evaporation [8] using a sweeped radio-frequency field $\nu_{\mathrm{rf}}$ which allows us to adjust the temperature of the atomic sample. The field induces an adiabatic transition from $m_{F}=4$ to $m_{F}=-4$ at a position depending on $\nu_{\mathrm{rf}}$. This allows one to eject selectively the high energy tail of the atomic distribution. The frequency $\nu_{\mathrm{rf}}$ is ramped down linearly in $10 \mathrm{~s}$ from $4 \mathrm{MHz}$ to an adjustable final value. The smallest temperature for the present data is $7 \mu \mathrm{K}$, obtained for $\nu_{\mathrm{rf}}=700 \mathrm{kHz}$ and $N=0.76 \times 10^{6}$. Once the cloud is prepared at the desired temperature, the rf field is turned off and the atoms remain in the dark for a variable relaxation time, ranging between 0 and $300 \mathrm{~s}$, after which we measure the number of remaining atoms and their temperature.

The detection is made by an absorption imaging technique. The magnetic trap is switched off in $300 \mu \mathrm{s}$, while a $0.1 \mathrm{mT}$ magnetic field directed along $x$ is applied. During this switching-off, the atomic magnetic moments follow adiabatically the local direction of the field at the center of the trap and end up aligned with the $x$ axis. A $100 \mu$ s pulse from a weak $\left(30 \mu \mathrm{W} \mathrm{cm}^{-2}\right)$ circularly polarized probe beam resonant with the closed transition $\left|6 S_{1 / 2}, F=m_{F}=4\right\rangle \rightarrow\left|6 P_{3 / 2}, F=m_{F}=5\right\rangle$ and propagating along $x$ illuminates the atomic cloud. A 1:1 optical system images the probe beam with the atomic cloud onto a CCD array. A second image with no atoms is taken $200 \mathrm{~ms}$ later to determine the laser intensity profile. The logarithm of the ratio of the two images then yields the cloud's optical density.

In this way we have access to the column density $\int n(\mathbf{r}) d x$, where $n(\mathbf{r})$ is the spatial density. Assuming a Gaussian spatial distribution with a cylindrical symmetry around $y$, we determine the number $N$ of atoms in the cloud and its temperature $T$. This measurement is destructive, so we repeat the procedure for the same initial conditions and for various relaxation times. The rms fluctuations are $\sim 5 \%$ for the initial atom number and $\sim 2 \%$ for the initial temperature.

Typical time evolutions for $N$ and $T$ are shown in Figs. 2(a) and 2(b). The evolution for $N(t)$, shown in logarithmic scale, clearly exhibits a nonexponential behavior which is a signature of a multibody decay process. The decrease of $N$ is accompanied by an increase of $T$ that can be simply understood. Any inelastic loss process occurs preferentially at the center of the trap where the atomic density is maximum. The atoms undergoing an inelastic collision therefore have a belowaverage potential energy and the elimination of these atoms leads to a relaxation heating of the cloud [8].

For a more quantitative analysis, we now assume that the nonexponential decay is caused by a two-body inelastic process with a cross section $\sigma_{\text {in }}=\beta(v) / v$, where $v$ is the relative velocity of the two colliding

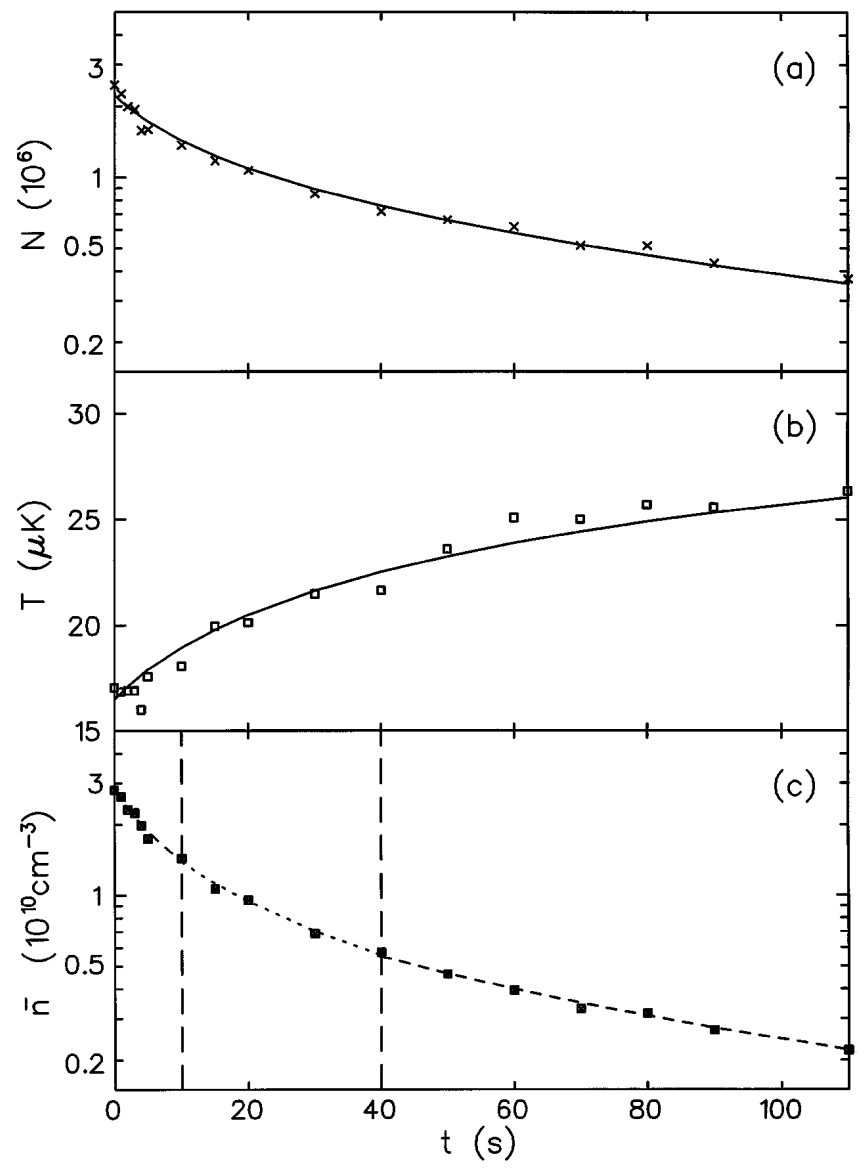

FIG. 2. (a) Total number of atoms remaining in the trap after a relaxation time $t$, exhibiting a nonexponential decay. (b) Temperature and (c) density evolution of the atomic sample. In each of the three intervals indicated in (c), the data are fitted with Eq. (2) (dashed and dotted lines). The solid curves in (a) and (b) are solutions of Eqs. (1) with $G(T) \propto T^{-0.63}$. 
particles [9]. When $v$ tends to zero, the rate coefficient $\beta(v)$ is known to have a finite nonzero limit [10].

We put $q \times(3+3) k_{B} T$ for the average energy removed from the harmonic trap when two atoms escape after an inelastic collision. The constant $q$ accounts for the below-average potential energy mentioned above. When $\beta(v)$ varies over the thermal distribution, $q$ also accounts for the difference between the average kinetic energy of each colliding particle and $(3 / 2) k_{B} T$. For instance, $\beta(v) \propto v^{-2 \nu}$ leads to $q=(9-2 \nu) / 12$. We obtain

$$
\frac{\dot{N}}{N}=-\alpha-G \bar{n}, \quad \frac{\dot{T}}{T}=(1-q) G \bar{n},
$$

where $\bar{n}=\int n^{2}(\mathbf{r}) d^{3} r / \int n(\mathbf{r}) d^{3} r=n(\mathbf{0}) / 2 \sqrt{2}$ is the average density in the trap and $G(T)$ is the two-body rate coefficient which can be calculated from $\beta(v)$. The coefficient $\alpha$ describes losses from the trap due to collisions with the residual background gas. Using $\bar{n} \propto N T^{-3 / 2}$ we get $\dot{\bar{n}}=-\alpha \bar{n}-\tilde{G} \bar{n}^{2}$, where $\tilde{G}=[1+$ $\left.\frac{3}{2}(1-q)\right] G$.

In the simple case $\beta(v)=\beta_{0}$ one finds $q=3 / 4$, $\beta_{0}=G=(8 / 11) \tilde{G}$, and

$$
\bar{n}(t)=\bar{n}_{0} e^{-\alpha t}\left[1+\left(1-e^{-\alpha t}\right) \tilde{G} \bar{n}_{0} / \alpha\right]^{-1} .
$$

Initially, we tried to fit with this function each data set giving the time evolution of $\bar{n}$. We found a $\tilde{G}$ depending on the initial temperature of the data set. This is not consistent with the hypothesis $\beta(v)=\beta_{0}$. Consequently, we have used the following procedure to extract $\tilde{G}(T)$ from the experimental data.

We first divide each data set into three time intervals during which the temperature is constant to within $\pm 10 \%$ with a mean value $T_{i}(i=1,2,3)$ [Fig. 2(c)]. This ensures that also $\tilde{G}$ changes little within each time interval. We then perform a least-squares fit of the evolution of $\bar{n}(t)$ over each interval with Eq. (2), using $\bar{n}_{0}$ and $\tilde{G}\left(T_{i}\right)$ as fit parameters [11]. The dashed and dotted curves in Fig. 2(c) are examples of the best fits in the three time intervals. The parameter $\alpha$ is adjusted to $\alpha^{-1}=200 \mathrm{~s}$ by comparing the decay rate of samples with equal temperatures and different densities.

Figure 3 shows the results of this analysis. The vertical error bars give the statistical error of $\tilde{G}$ determined from the least-squares fits. The horizontal error bars give the standard deviation of the temperature in the considered time interval. In double log scale the points are distributed along a straight line, and a linear fit gives $\tilde{G}(T)=2.4 \times 10^{-11} T^{-0.63} \mathrm{~cm}^{3} \mathrm{~s}^{-1}$, where $T$ is in $\mu \mathrm{K}$.

The power-law dependence $\tilde{G}(T) \propto T^{-\nu}$ implies $\beta(v) \propto v^{-2 \nu}$ on the microscopic level. With $\nu=0.63$ one gets $q=0.65$ and the final result is [12]

$$
G(T)=(1.5 \pm 0.3 \pm 0.3) \times 10^{-11} T^{-0.63} \mathrm{~cm}^{3} \mathrm{~s}^{-1} .
$$

The first error is statistical and estimated from the fit in Fig. 3, whereas the second error represents systematic uncertainties in the measurement of the atomic density.

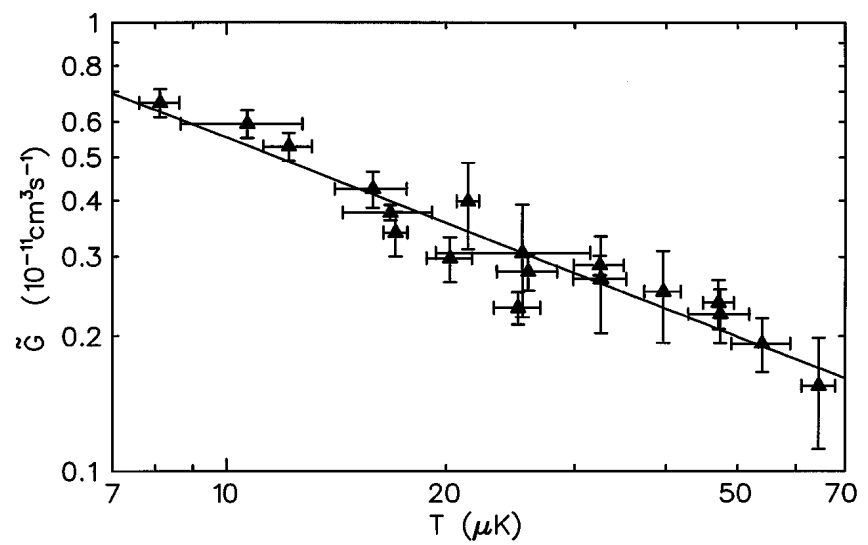

FIG. 3. Temperature dependence of the rate coefficient $\tilde{G}$. Each point is derived from a fit to $\bar{n}(t)$ [see Fig. 2(c)]. The straight line is a fit with a power law, giving $\tilde{G} \propto T^{-0,63}$.

The statistical error on the exponent $\nu=0.63$ is $10 \%$. Typical values for the elastic collision rate are $\gamma_{e} \approx$ $10 \mathrm{~s}^{-1}$, much larger than the inelastic collision rates $\left(G \bar{n}<0.1 \mathrm{~s}^{-1}\right)$, which justifies our assumption of thermal equilibrium.

The measured value of the two-body rate coefficient $G$ is as high as $4 \times 10^{-12} \mathrm{~cm}^{3} \mathrm{~s}^{-1}$ for $8 \mu \mathrm{K}$. This rate is of the same order as the one measured for unpolarized atomic samples prepared in the $F=4$ ground state [13]. However, in the latter case such a large rate is easily accounted for by exchange collisions. We have therefore performed a similar set of experiments at a current of only $13.8 \mathrm{~A}$ in the trap coils. In this case, the atomic sample is always fully polarized because only atoms in the substate $F=m_{F}=4$ can be held against gravity. In the temperature range 5-20 $\mu \mathrm{K}$, the measurements in this "weak" trap lead to values for $\tilde{G}$ either in agreement or even higher than the ones plotted in Fig. 3. We attribute the deviation to residual evaporation out of the shallow trap (calculated depth $\sim 30 \mu \mathrm{K}$ ) that leads to an extra density-dependent loss term.

In addition, we tried to detect atoms in the substates $F=4, m_{F} \neq 4$ after various relaxation times, by taking an image directly after having lowered the trap current from 51 to $13.8 \mathrm{~A}$ in $25 \mathrm{~ms}$. Because of their different magnetic moments, atoms in the various substates fall out of the trap in successive bunches. We did not find any atoms in the $m_{F} \neq 4$ substates, whereas in a deliberately depolarized sample we were able to detect a 5\% fraction of the atoms in $m_{F}=3$. This confirms that nearly all of the atoms are lost via hyperfine-changing collisions.

We now return to the variations of $N(t)$ and $T(t)$ given in Fig. 2, which we model using $G(T)=g_{0} T^{-0.63}$ and the analytical solutions of (1). For this data set, the best fit is obtained for $g_{0}=1.2 \times 10^{-11} \mathrm{~cm}^{3} \mathrm{~s}^{-1}$, in good agreement with Eq. (3). The results are indicated with solid lines in Figs. 2(a) and 2(b). Our model clearly gives a quantitative account for both the decrease of $N$ and 
the increase of $T$. This is the case for the six data sets considered here, except for the one with the lowest initial temperature. For this data set the temperature increase is larger than predicted by $\sim 50 \%$. The reason may be smallangle collisions between the trapped atoms and the fast atoms created during inelastic cold collisions, the coldest cloud being the most sensitive to this extra-heating term.

The present results for the two-body decay rate $G$ are much higher than the values measured or predicted for other cold alkali metals such as lithium, sodium or rubidium in their doubly polarized state. Those values are all in the range $(6-20) \times 10^{-15} \mathrm{~cm}^{3} \mathrm{~s}^{-1}$ [14-16]. The values predicted a few years ago for a cold gas of doubly polarized $\mathrm{Cs}$ atoms were also in that same range [4]. The discrepancy of 3 orders of magnitude between those predictions and the present results can be explained in two complementary fashions.

First, the zero-energy resonance recently observed for cold Cs elastic collisions [6] is a signature for a bound or virtual state very close to the dissociation limit of the Cs-Cs triplet potential. This leads to a large $s$-wave scattering length $a(|a|>140 \AA[6,17])$. It also enhances strongly the occupation probability at short relative distances for the low energy collisional states of two Cs atoms. Hence, the rate for any two-body inelastic process will be large. Furthermore, $\beta(v)$ is expected to increase for decreasing $v$ until it saturates for $\hbar / m v>|a|$, i.e., $T<5 \mu \mathrm{K}$. This qualitative argument is in accordance with the measured variation of $G(T)$ over the studied temperature range.

Second, there exists, in addition to the standard dipoledipole interaction, an extra spin-spin coupling, arising as a second-order effect in the electronic spin-orbit coupling [18] that was not included in [4]. Recent calculations including this extra term lead to predictions of the same order as the observed decay rates [19].

Finally, we note that in our experiments the ratio between the thermalization rate due to elastic collisions $\gamma_{e} / 10$ [6] and the loss rate due to collisions with the background gas is very favorable $(\sim 200)$. Indeed, for an evaporation leading to quantum degeneracy, a factor larger than 50 is sufficient [20]. Nevertheless, the highest phase space density we could attain was only $\sim 10^{-5}$ for a cloud at $4 \mu \mathrm{K}$. This limitation originates from the large spin relaxation rate which at $10 \mu \mathrm{K}$ is only a factor of 10 below the thermalization rate. The achievement of BEC with cesium atoms in this doubly polarized state seems therefore very unrealistic [21].

We are grateful to M. Arndt, M. Ben Dahan, and O. Verzelen for their assistance and to P. Julienne, C. Cohen-Tannoudji, C. Salomon, H. Stoof, and the LKB group for valuable discussions. J.S. acknowledges sup- port by the Alexander von Humboldt Foundation. This work was partially supported by CNRS, Collège de France, DRET, DRED, and EC (TMR network ERB FMRX-CT96-0002).

*Dipartimento di Fisica, Universitá di Pisa, Piazza Torricelli 2, I-56100 Pisa, Italy.

†Unité de recherche de l'ENS et de l'Université Pierre et Marie Curie, associée au CNRS.

[1] M. H. Anderson et al., Science 269, 1989 (1995).

[2] C. C. Bradley, C. A. Sackett, and R. G. Hulet, Phys. Rev. Lett. 78, 985 (1997); see also C. C. Bradley et al., Phys. Rev. Lett. 75, 1687 (1995).

[3] K. B. Davis et al., Phys. Rev. Lett. 75, 3969 (1995).

[4] E. Tiesinga et al., Phys. Rev. A 46, R1167 (1992); E. Tiesinga, B. J. Verhaar, and H. T. C. Stoof, Phys. Rev. A 47, 4114 (1993).

[5] Similar measurements are currently performed in Oxford [C. Foot (private communication)].

[6] M. Arndt et al., Phys. Rev. Lett. 79, 625 (1997).

[7] A. Steane et al., Phys. Rev. Lett. 74, 4972 (1995).

[8] O.J. Luiten, M.W. Reynolds, and J.T.M. Walraven, Phys. Rev. A 53, 381 (1996).

[9] We performed a similar analysis assuming a three-body decay process and found that it is not at all consistent with the data.

[10] See, e.g., L.D. Landau and E. M. Lifshitz, Quantum Mechanics (Pergamon Press, Oxford, 1977), Sect. 143.

[11] The error incurred by this step approximation for $\tilde{G}(T)$ is negligible compared to the statistical errors.

[12] One also finds $G(T)=\Gamma(3-2 \nu / 2) \beta\left(\sqrt{k_{B} T / m}\right) /$ $2^{2 \nu-1} \sqrt{\pi}$

[13] P. Lemonde et al., Europhys. Lett. 32, 555 (1995).

[14] A. J. Moerdijk and B.J. Verhaar, Phys. Rev. A 53, R19 (1996).

[15] H. Boesten, A. J. Moerdijk, and B. J. Verhaar, Phys. Rev. A 54, R29 (1996).

[16] C. J. Myatt et al., Phys. Rev. Lett. 78, 586 (1997).

[17] Recent photo-association measurements in a Cs MOT confirm this lower bound [P. Pillet (private communication)].

[18] P. Julienne, J. Mol. Spectrosc. 56, 270 (1975).

[19] P. Leo, E. Tiesinga, P. Julienne, and T. Walker (private communication). These calculations extrapolate dipolar relaxation rates measured at room temperature by N.D. Bhaskar et al. [Phys. Rev. Lett. 44, 930 (1980)].

[20] C. R. Monroe et al., Phys. Rev. Lett. 70, 414 (1993).

[21] We have started to investigate with the same setup the evaporative cooling of a cesium gas magnetically trapped in the $F=3, m=-3$ state. Temperatures as low as $50 \mathrm{nK}$ have been achieved, with a final phase space density $\sim 10^{-2}$ [D. Guéry-Odelin, J. Söding, P. Desbiolles, and J. Dalibard, Opt. Express (to be published)]. The analysis of the experimental limitations to the cooling of Cs in this lower hyperfine state is currently under way. 